\title{
Incidental detection of COVID-19 associated pneumonia by thyroid scintigraphy
}

Mehrosadat Alavi ${ }^{1,2}$, Fariba Jafari'10

'Department of Nuclear Medicine, Medicine School, Shiraz University of Medical Sciences, Shiraz, Iran

Ilonizing and Nonlonizing Radiation Protection Research Center (INIRPRC), Shiraz University of Medical Sciences., Shiraz, Iran

[Received 14 II 2021; Accepted 15 III 2021]

\begin{abstract}
This report presents a case of a 49-year-old woman with complaint of sore throat and front neck pain, who referred to a hospital for thyroid scan due to suppressed TSH level $(0.005 \mathrm{mU} / \mathrm{L})$. Diffuse and bilateral lungs uptake in the scan was noticed incidentally. The patient had positive history of covid-19 symptoms. Multifocal and bilateral ground-glass opacities (GGOs) in both lungs were compatible with typical features of lung involvement in COVID-19-associated pneumonia.
\end{abstract}

KEY words: COVID-19; pneumonia; thyroid scintigraphy

Nucl Med Rev 2021; 24, 2: 113-114

A 49-year-old female was presented with complaints of sore throat and front neck pain for 1 month. She referred to the nuclear medicine department to perform a thyroid scan. The thyroid gland was tender on physical examination. Also, the laboratory assay showed suppressed TSH level (0.005 mU/L). 15 minutes after
Intravenous injection of $185 \mathrm{MBq}\left[{ }^{99 \mathrm{~m}} \mathrm{Tc}\right]$ pertechnetate, an anterior planar image of the neck was obtained. The scan revealed diffusely decreased radiotracer uptake throughout the thyroid with poor delineation of the thyroid gland which was suggestive of subacute thyroiditis (Fig. 1). Moreover, significant, diffuse and bilateral [99mTc]
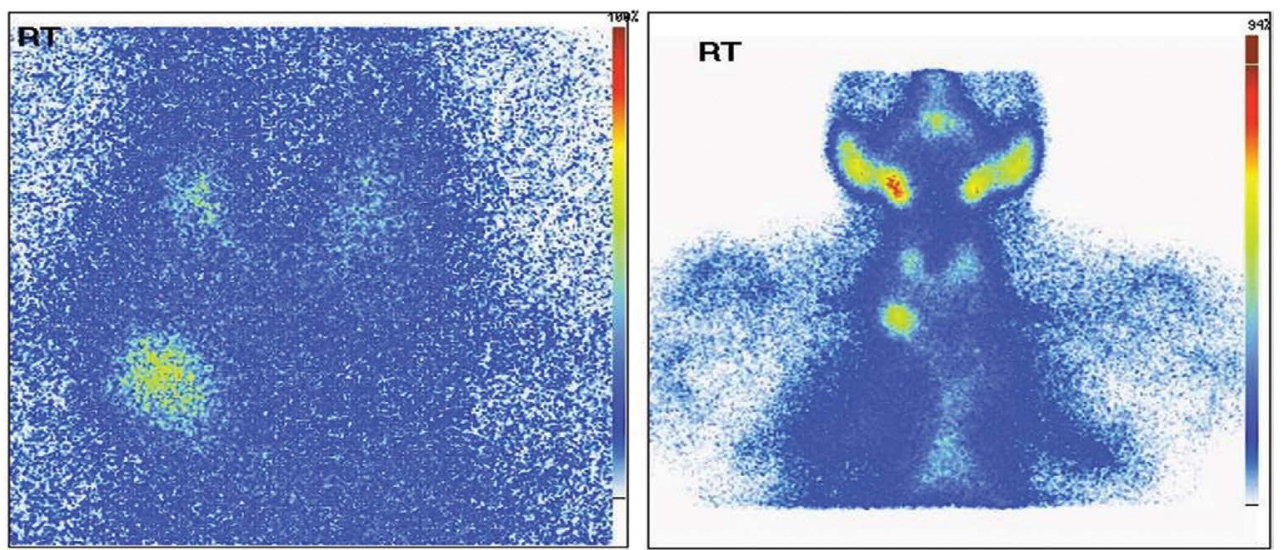

Figure 1. [99mTC] pertechnetate thyroid scan. Anterior planar image of the neck revealed diffusely decreased radiotracer uptake throughout the thyroid with poor delineation of the thyroid gland and decreased thyroid to background ratio. Also, significant, diffuse and bilateral uptake in both lungs is noted 


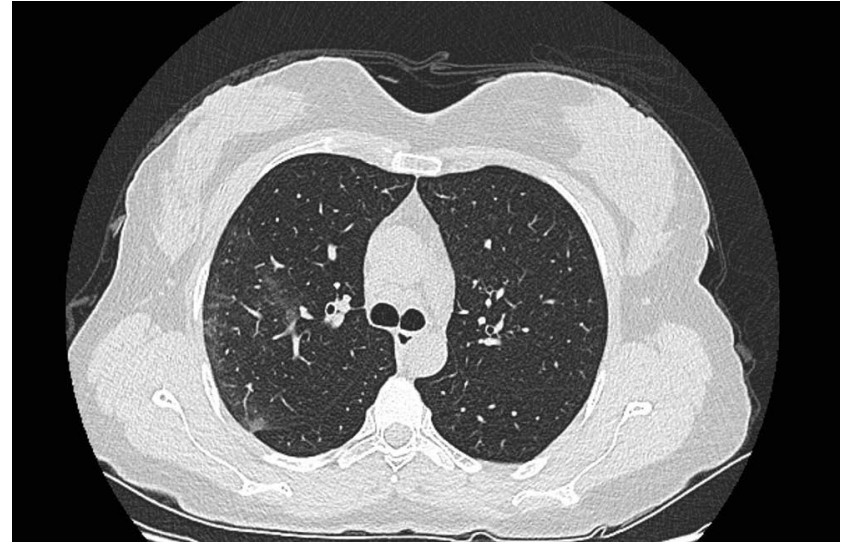

Figure 2. Transaxial chest HRCT scan showed bilateral and multifocal patchy ground-glass opacities (GGOs), (more in the right lung), predominantly located in the peripheral of the chest

pertechnetate in both lungs fields caught our attention. After that, the patient was asked about experiencing any infectious symptoms. She had fever, cough, dyspnoea and myalgia for the past 40 days, which were typical symptoms of COVID-19 pneumonia [1, 2]. The patient underwent a chest high-resolution CT scan (HRCT) that revealed multifocal and bilateral ground-glass opacities (GGOs), predominantly distributed in the peripheral. These findings were compatible with typical findings of COVID-19-associated pneumonia (Fig. 2) [3, 4]

COVID-19 disease causes severe acute respiratory syndrome. SARS-CoV-2 was first recognized at the end of 2019 and became a global concern very soon [5]. Nowadays, many people around the world have experienced a range of clinical manifestation, from no symptoms to critical illness. The purpose of this presentation is to spread awareness among nuclear medicine physicians to develop their knowledge about incidental detection of COVID-19 disease in patients who undergo routine SPECT/CT or PET/CT scans during this pandemic, especially in high COVID-19 prevalence areas [6-8].

\section{Conflict of interest}

The authors declare that they do not have any conflict of interest.

\section{References}

1. Huang C, Wang Y, Li X, et al. Clinical features of patients infected with 2019 novel coronavirus in Wuhan, China. Lancet. 2020; 395(10223): 497-506, doi: 10.1016/S0140-6736(20)30183-5, indexed in Pubmed: 31986264

2. Jiang F, Deng $L$, Zhang $L$, et al. Review of the clinical characteristics of coronavirus disease 2019 (COVID-19). J Gen Intern Med. 2020; 35(5): 1545-1549, doi: 10.1007/s11606-020-05762-W, indexed in Pubmed: 32133578.

3. Ali SA, Abdelkawi MM. Incidentally recognized COVID-19 pneumonia in routine oncologic 18F-FDG PET/CT examinations: a local experience during pandemic era. Egyptian Journal of Radiology and Nuclear Medicine. 2020; 51(1), doi: 10.1186/s43055-020-00333-9.

4. Fang $\mathrm{Y}$, Zhang $\mathrm{H}$, Xie J, et al. Sensitivity of chest CT for COVID-19: comparison to RT-PCR. Radiology. 2020; 296(2): E115-E117, doi: 10.1148/radiol.2020200432, indexed in Pubmed: 32073353.

5. Zhou $P$, Yang $X L$, Wang $X G$, et al. A pneumonia outbreak associated with a new coronavirus of probable bat origin. Nature. 2020; 579(7798): 270-273, doi: 10.1038/s41586-020-2012-7, indexed in Pubmed: 32015507

6. Tulchinsky M, Fotos JS, Slonimsky E. Incidental CT findings suspicious for covid-19-associated pneumonia on nuclear medicine examinations: recognition and management plan. Clin Nucl Med. 2020; 45(7): 531-533, doi: 10.1097/RLU.0000000000003100, indexed in Pubmed: 32502091.

7. Hindle-Katel W, Oen-Hsiao J, Lussnig E, et al. Incidental finding of COVID-19 pulmonary infiltrates on SPECT/CT attenuation correction CT. J Nucl Cardiol. 2020; 27(4): 1385-1386, doi: 10.1007/s12350-020-02178-1, indexed in Pubmed: 32394407

8. Cohen F, Chiche L, Rebaudet S, et al. Incidental pneumonia on PET imaging . Rev Med Interne. 2021; 42(3): 225-226, doi: 10.1016/j.revmed.2020.09.003, indexed in Pubmed: 33160705. 